Pacific Journal of Mathematic

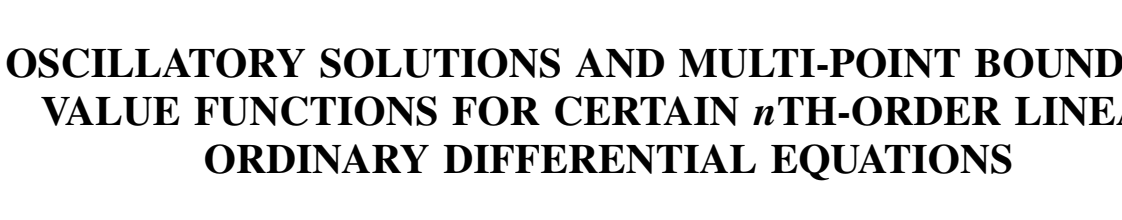




\title{
OSCILLATORY SOLUTIONS AND MULTI-POINT BOUNDARY VALUE FUNCTIONS FOR CERTAIN $n$ th-ORDER LINEAR ORDINARY DIFFERENTIAL EQUATIONS
}

\author{
M. S. KEENER
}

Consider the $n$th order linear differential equation

$$
y^{(n)}+\sum_{k=0}^{n-1} p_{k}(t) y^{(k)}=0,
$$

where $p_{k}(t) \in C[\alpha, \infty)$. This study explores some of the relationships between multi-point boundary value functions for (1) and the character of oscillatory solutions of (1). In particular, it is supposed for $(1)$ that a certain $(n-1)$ point boundary value problem has no nontrivial solution and that two nontrivial solutions with $(n-1)$ zeros in common are constant multiples of each other. Under these conditions it is shown that there exists an integer $i, 1 \leqq i \leqq n-1$, such that for each $a>\alpha$ and every integer $l, 1 \leqq l \leqq i-1$, there is an oscillatory solution of (1) with a zero of exact multiplicity $l$ at $t=a$. Furthermore, any solution of (1) with a zero at $t=a$ of multiplicity $l \geqq i$ is nonoscillatory.

In general, simple examples illustrate that oscillatory behavior varies widely under no additional conditions on the equation (1). In order to give some structure on which to base an investigation of the given equation, we employ multi-point boundary value functions. These functions have been studied by Alieu (in papers unavailable to the author) and by A. C. Peterson ([6], [7]). These functions were essentially used by Hanan [3] for $n=3$ and, for $n=4$, by Leighton and Nehari [5] as well as the author [4]. The results in this paper generalize some of the ideas of these papers.

We shall need the following definitions.

DEFINITION. We say a nontrivial solution $y(t)$ of (1) has an $i_{1}-i_{2}-\cdots-i_{k}, \sum_{j=1}^{k} i_{j}=n$, distribution of zeros on an interval $I$ provided there exists points $t_{1}<t_{2}<\cdots<t_{k}$ in $I$ such that $y(t)$ has a zero of order at least $i_{j}$ at the point $t=t_{j}, j=1,2, \cdots, k$. For each $t \in[\alpha, \infty), r_{i_{1} i_{2} \cdots i_{k}}(t)$ denotes the infimum of the set of numbers $b>t$ for which there exists a nontrivial solution $y(t)$ of (1) with an $i_{1}-i_{2}-\cdots-i_{k}$ distribution of zeros on $[t, b]$. If no such distribution exists, we write

$$
r_{i_{1} i_{2} \cdots i_{k}}(t)=\infty .
$$

If $r_{i_{1} i_{2} \cdots v_{k}}(t)=\infty$ for all $t \in[\alpha, \infty)$, we write 


$$
r_{i_{1} i_{2} \cdots i_{k}}=\infty
$$

The condition on equation (1) we employ in this paper is

$$
r_{11 \cdots 1 \cdot 2 \cdot \cdots 1}=\infty \text {, }
$$

where the number two in the subscript appears in the $j$ th position for some $1 \leqq j \leqq n-1$. The position of this number two proves to be important. In order to simplify the statement of lemmas, theorems, etc., we introduce the following notation. define

Notation. For integers $i$ and $j, 1 \leqq i \leqq n-2,1 \leqq j \leqq n-i$,

$$
S_{(n-i) j}(t) \equiv r_{11 \cdots 1(i+1) \cdots 1}(t),
$$

where the number $(i+1)$ in the above subscript appears in the $j$ th position. The symbol $S_{(n-i) j}(t)$ denotes then the infimum of the set of number $b>t$ for which there exists a nontrivial solution $y(t)$ with $(n-i)$ distinct zeros at points $t_{i}, t \leqq t_{1} \leqq<t_{2}<\cdots<t_{n-i} \leqq b$ and a zero at $t=t_{j}$ of order at least $(i+1)$. As before, if no such solution $y(t)$ exists, we write

$$
S_{(n-i) j}(t)=\infty ;
$$

if $S_{(n-i) j}(t)=\infty$ for all $t \in[\alpha, \infty)$, we write

$$
S_{(n-i) j}=\infty \text {. }
$$

In this notation condition (2) above is simply

$$
S_{(n-1) j}=\infty \text {. }
$$

For $n=3$, Hanan [3] has studied the oscillatory properties of equations with $S_{21}=\infty$ or $S_{22}=\infty$. Dolan [1] has studied third-order equations for which $S_{21}(t)<S_{22}(t)$. For $n=4$, Leighton and Nehari [5] considered the equation

$$
\left[r(x) y^{\prime \prime}\right]^{\prime \prime}-p(x) y=0,
$$

$r(x), p(x) \in C[\alpha, \infty), r(x)$ and $p(x)$ are positive on $[\alpha, \infty)$. They show that $S_{32}=\infty$ for such equations. The author [4] has considered fourth-order equations for which $S_{31}=\infty$ or $S_{33}=\infty$. The methods of these authors are difficult to generalize since they make use of the relatively low degree of the equation in question.

In this paper we make use of the following standing hypothesis:

$(H)$ Any two nontrivial solutions with $(n-1)$ zeros in common are constant multiples of each other.

The hypothesis seems to play a fundamental role in studies of 
this kind. For $n=3, S_{21}=\infty$ or $S_{22}=\infty$ imply $(\mathrm{H})$ trivially. For $n=4$, added conditions are often used to guarantee that $(\mathrm{H})$ is satisfied (see [4], [5]). For $n>4$, little seems to be known about equations satisfying $(\mathrm{H})$. However, assuming $S_{(n-1) j}=\infty$ for some integer $j, 1 \leqq j \leqq n-1$, it is clear that two nontrivial solutions with $(n-1)$ distinct zeros in common are constant multiples of each other. Furthermore, under the same hypothesis, $(\mathrm{H})$ will hold provided $(n-1) \times n$ matrices of the form

$$
\left(z_{1}^{\left(m_{i}\right)}\left(a_{\imath}\right), z_{2}^{\left.\prime m_{i}\right)}\left(a_{i}\right), \cdots, z_{n}^{\left(m_{i}\right)}\left(a_{\imath}\right)\right)
$$

$\left(i=1, \cdots, k ; 0 \leqq m_{\imath} \leqq \mu_{\imath}, \sum_{i=1}^{k} \mu_{\imath}=n-1 ; 2 \leqq k<n-1\right)$ have rank $(n-1)$. Here $\left\{z_{i}(t)\right\}_{i=1}^{\infty}$ is a fundamental set of solutions for equation 1. This latter condition guarantees that solutions exist satisfying $(n-2)$ homogeneous conditions and one inhomogeneous condition. The proof that $S_{(n-1) j}=\infty$ implies (H) follows then by an elementary argument. We shall assume $(\mathrm{H})$ throughout this study.

Peterson [6] has shown that if $r_{111 \ldots 11}(t)<\infty$ for some $t \in[\alpha, \infty)$, then there exists at most one integer $j, 1 \leqq j \leqq n-1$, for which $S_{(n-1) j}(t)=\infty$. Assuming $(\mathrm{H})$, we show in this paper that if $S_{(n-1) j}=\infty$ for equation (1) and $a \in(\alpha, \infty)$, then for each integer $l, 1 \leqq l \leqq j$, there exists an oscillatory solution of (1) with a zero of exact order $l$ at $t=a$.

The following lemmas prove to be quite useful in the construction of solutions with certain types of distributions of zeros. Lemma 1 is due to T. Sherman [8], while Lemma 2 has been used by a number of authors. Lemma 3 and Lemma 4 are exercises stated here for reference purposes.

Lemma 1. (Sherman) Let $f$ and $g$ be functions with the following properties:

(i) $g$ has a zero of order $q \geqq 0$ at $t=a$ and $g^{(q)}(a)>0$;

(ii) $f$ has a zero of order $p>q$ at $t=a$ and $f^{(p)}(a)>0$;

(iii) $f(t) g(t) \neq 0$ for $t \in(a, b]$;

(iv) $f, g \in C^{p}[a, b]$.

Then given any $K>0$ there is a constant $c, 0<c<K$, such that $h(t)=f(t)-c g(t)$ has a simple zero at some point $t_{c} \in(a, b]$ and $h(t) \neq 0$ for $t \in\left(t_{c}, b\right]$.

LEMMA 2. Let $f$ and $g$ be functions with the following properties:

(i) $f$ has a zero of order $n \geqq 1$ at $t=a$ and a zero of order $m \geqq 1$ at $t=b$;

(ii) $g$ has a zero of exact order $n_{1}<n$ at $t=a$ and $a$ zero of exact order $m_{1}<m$ at $t=b$;

(iii) $f(t) g(t) \neq 0$ for $t \in(a, b)$; 
(iv) $f, g \in C^{p}[a, b]$, where $p=\max \{n, m\}$. Then there exists a nontrivial linear combination $h(t)$ of $f$ and $g$ such that $h(t)$ has a double zero in $(a, b)$.

LEMMA 3. Let $f$ and $g$ be functions with the following properties:

(i) $f$ has a zero of even multiplicity at $t=a$.

(ii) $g(t) \neq 0$ in some interval I containing $t=a$.

(iii) $f$ and $g$ are continuous and positive on $I \backslash\{a\}$.

Then there exists a constant $K>0$ such that for $k \in(0, K)$ the function $h(t)=f(t)-k g(t)$ has at least two distinct zeros of odd order on $I$.

LEMMA 4. Let $f$ and $g$ be functions with the following properties:

(i) $f$ has an isolated zero of odd order at $t=a$;

(ii) $g$ does not vanish in some interval I containing the point $t=a$;

(iii) $f, g \in C(I)$.

Then there exists a constant $K>0$ such that if $|k|<K$, the function $h(t)=f(t)-k g(t)$ has at least one zero of odd order on the interval $I$.

LEMMA 5. Let $f$ and $g$ be functions with the following properties:

(i) $g$ has a zero of order $q \geqq 0$ at $t=a$ and $(-1)^{q} g^{(q)}(a)>0$;

(ii) $f$ has a zero of order $p>q$ at $t=a$ and $(-1)^{p} f^{(p)}(a)>0$;

(iii) $f(t) \cdot g(t) \neq 0$ for $t \in[b, a)$;

(iv) $f, g \in C^{p}[b, a]$.

Then given any $K>0$ there exists a constant $c, 0<c<K$, such that $h(t)=f(t)-c g(t)$ has a simple zero at some point $t_{c} \in(b, a)$ and $h(t) \neq 0$ for $t \in\left[b, t_{c}\right)$.

Lemma 5 is an analogue of Lemma 1 and may be proved by applying Lemma 1 to the functions $F(x)=f(a-x)$ and $G(x)=g(a-x)$ on the interval $[0, a-b]$.

The theorem below provides a good illustration of the techniques used in the construction of solution with certain desired zero distributions. The technique is used often throughout the paper. Furthermore, it gives a relationship between an $(n-1)$ point disconjugacy criteria and a two point disconjugacy criteria. The latter criteria has received more attention than the former with regards to conditions on the coefficients of (1) to induce such a disconjugacy. If $n=4$, Theorems 2 and 3 are similar to Lemmas 2.1 and 2.3 in [5].

THEOREM 1. If $S_{(n-1) 2}=\infty$, then $S_{21}(t)=\infty$ for all $t>\alpha$.

To prove the theorem it is necessary and sufficient to show that the solution $y(t)$ given by 


$$
\begin{aligned}
y^{(p)}(a) & =0, \quad p=0,1, \cdots, n-2, \\
y^{(n-1)}(a) & =1,
\end{aligned}
$$

$(\alpha<a)$ does not vanish on $(a, \infty)$. Suppose to the contrary that $y(b)=0$ for some $b>a$. We will show that there exists a nontrivial solution $w(t)$ with $(n-1)$ distinct zeros and a zero of multiplicity two in the second position.

We first show that there exists a solution $y_{1}(t)$ with a zero of order $(n-2)$ at $t=a$ and two distinct zeros, of odd multiplicity. Let $x_{1}(t)$ denote a nontrivial solution of (1) satisfying the following conditions:

$$
\begin{aligned}
x_{1}^{(p)}(a) & =0, \quad p=0,1,2, \cdots, n-3 ; \\
x_{1}(b) & \neq 0 .
\end{aligned}
$$

Note that $x_{1}^{(n-2)}(a) \neq 0$ since $x_{1}(t)$ and $y(t)$ are not constant multiples of each other. If $y^{\prime}(b)=0$, we may choose $\operatorname{sgn} x_{1}(b)$ so that Lemma 3 will apply at the point $t=b$. In this way, we will have a nontrivial solution with the desired properties. If $y^{\prime}(b) \neq 0$, we may choose $x(t)$ so that $x_{1}^{(n-2)}(a)>0$ and Lemma 1 will apply. By applying Lemma 4 at the point $t=b$ and then applying Lemma 1 , we have the desired solution. In either case, the solution $y_{1}(t)$ exists with two zeros of odd multiplicity at $t=b_{1}<\bar{b}_{2}$.

Now we show there exists a solution $y_{2}(t)$ with a zero of order $(n-3)$ at $t=a$ and three distinct zeros on $(a, \infty)$, one of which is of odd multiplicity. Let $x_{2}(t)$ denote a solution of (1) satisfying the following conditions:

$$
\begin{aligned}
x_{2}^{(p)}(a) & =0, \quad p=0,1, \cdots, n-4 ; \\
x_{2}\left(b_{1}\right) & =0 ; \\
x_{2}\left(\overline{b_{2}}\right) & \neq 0 .
\end{aligned}
$$

Now we apply Lemma 4 at the point $t=\bar{b}_{2}$ to obtain a constant $K>0$ such that if $0<|k|<K$,

$$
y_{1}(t)-k x_{2}(t)
$$

has a zero of odd multiplicity on $\left(b_{1}, \infty\right)$. We may choose $\operatorname{sgn} x_{2}^{(n-3)}(a)$ so that Lemma 1 applies, and hence there exists a number $k, 0<$ $|k|<K$, such that

$$
y_{2}(t)=y_{1}(t)-k x_{2}(t)
$$

has a simple zero at $t=b_{2} \in\left(a, b_{1}\right)$ and vanishes at $t=\bar{b}_{3} \in\left(b_{1}, \infty\right)$.

Similarly there exists a solution $y_{3}(t)$ with a zero of order $(n-4)$ at $t=a$ and four distinct zeros on $(a, \infty)$, at least one of which is 
of odd multiplicity. The procedure is as before except the solution $x_{3}(t)$ is chosen to satisfy the following $(n-1)$ conditions:

$$
\begin{aligned}
x_{3}^{(p)}(a) & =0, \quad p=0,1,2, \cdots, n-5 ; \\
x_{3}\left(b_{2}\right) & =0 ; \\
x_{3}\left(\bar{b}_{3}\right) & \neq 0 ; \\
x_{3}\left(b_{1}\right) & =0 .
\end{aligned}
$$

We may continue this process until we have obtained a nontrivial solution $y_{n-4}(t)$ such that $y_{n-4}(t)$ has a triple zero at $t=a$ and $(n-3)$ distinct zeros on $(a, \infty)$ at $t=b_{n-4}<\cdots<b_{1}<\bar{b}_{n-3}$ and a zero of odd multiplicity at $t=\vec{b}_{n-3}$. Let $x(t)$ denote a solution of (1) satisfying the follwing conditions:

$$
\begin{aligned}
x(a) & =x^{\prime}(a)=0 ; \\
x\left(b_{i}\right) & =0, \quad i=1,2, \cdots, n-4 ; \\
x\left(\bar{b}_{n-3}\right) & \neq 0
\end{aligned}
$$

where $\operatorname{sgn} x^{\prime \prime}(a)$ is chosen so that Lemma 5 applies at $t=a$. Then as above we apply Lemma 4 at the point $t=\bar{b}_{n-3}$ to obtain a constant $K>0$ such that if $0<|k|<K$,

$$
y_{n-4}(t)-k x(t)
$$

has $(n-3)$ distinct zeros on $(a, \infty)$. Corresponding to $K$, we apply Lemma 5 to obtain a nontrivial solution

$$
w(t)=y_{n-4}(t)-k x(t), \quad|k|<K,
$$

which has a simple zero on $(\alpha, a)$. Since $w(t)$ has the desired properties, we have $S_{(n-1) 2} \neq \infty$. This contradiction concludes the proof of the theorem.

An analogue of the method of proof used in Theorem 1 may be given to prove the following theorem. The primary alteration is the interchanging of the roles played by Lemma 1 and Lemma 5 .

THEOREM 2. If $S_{(n-1)(n-2)}=\infty$, then $S_{22}=\infty$ for all $t \in(\alpha, \infty)$.

The following theorem is fundamental in our work. The corollaries are along the lines of results of Peterson [7]. Basically the theorem provides us with the existence of certain nontrivial solutions with zeros of odd multiplicities which are useful in the constructions of certain solutions.

TheOREM 3. Suppose $S_{(n-1) j}=\infty$ for some integer $j, 1 \leqq j \leqq n-1$. Let $i$ and $k$ be integers, $1 \leqq i \leqq n-2,1 \leqq k \leqq n-i$. Suppose $y(t)$ 
is a nontrivial solution of $(1)$ with $(n-i)$ distinct zeros on $[a, b) \subset$ $[\alpha, \infty)$ at $a \leqq a_{1}<a_{2}<\cdots<a_{n-i}<b$ and $a$ zero at $t=a_{j}$ of order $(i+1)$.

(i) If there exists an integer $m$ for which either $a_{m}<a_{m+1}<a_{k}$ or $a_{k}<a_{m}<a_{m+1}$, then there exists a nontrivial solution $w(t)$ of (1) with $(n-i)$ distinct zeros on $[a, b), a$ zero in the $j$ th position of order $(i+1)$, and at least one zero on $\left(a, a_{k}\right) \cup\left(a_{k}, b\right)$ of odd multiplicity.

(ii) If $k \neq 1$, and $\alpha<a$, there exists a nontrivial solution $z(t)$ of $(1)$ with $(n-i+1)$ distinct zeros on $(\alpha, b)$, a zero in the $(k+1)$ st position of order $i$, and a zero of odd multipliplicity on $\left(\alpha, a_{k}\right)$.

(iii) If $k \neq n-i$, there exists a nontrivial solution $z(t)$ of (1) with $(n-i+1)$ distinct zeros on $[a, b)$, a zero of order $i$ in the kth position, and a zero of odd multiplicity on $\left(a_{k}, b\right)$.

To prove part (i) of the theorem, we assume $y(t)$ has a zero of even multiplicity at $t=a_{p}, p=1,2, \cdots, n-i$. Let $c \in[a, b)$ for which $y(c) \neq 0$ and $x(t)$ denote a nontrivial solution of (1) satisfying following boundary conditions:

$$
\begin{aligned}
x\left(a_{p}\right)=0, & p \neq a_{m}, a_{m+1} ; \\
x^{(p)}\left(a_{k}\right) & =0, \quad p=1,2, \cdots, i ; \\
x(c) & =0 .
\end{aligned}
$$

By $(\mathrm{H}), x\left(a_{m}\right) \times\left(a_{m+1}\right) \neq 0$ and so we may choose $x(t)$ so that $\operatorname{sgn} x\left(a_{m+1}\right)=$ $\operatorname{sgn} y\left(a_{m+1}+\varepsilon\right)$ for $\varepsilon>0$ and small. Let $I$ denote an interval containing $t=a_{m+1}$ for which $y(t)$ does not vanish on the set $I \backslash\left\{a_{m+1}\right\}$. Applying Lemma 3 , there exists a constant $K>0$ such that if $0<\lambda<K$,

$$
w(t)=y(t)-\lambda x(t)
$$

has two distinct zeros of odd mltiplicity on $I$. By (5) $w(t) \neq \equiv$ and the proof of part (i) is complete.

For the proof of part (ii), first suppose that $y(t)$ has a zero of odd multiplicity at $t=a_{1}$. Let $c \in[a, b)$ for which $y(c) \neq 0$ and $x(t)$ be a nontrivial solution of $(1)$ defined by the $(n-1)$ boundary conditions:

$$
\begin{aligned}
x\left(a_{p}\right) & =0, & p=2,3, \cdots, n-i ; \\
x^{(p)}\left(a_{k}\right) & =0, & p=1,2, \cdots, i-1 ; \\
x(c) & =0 . &
\end{aligned}
$$

By $(\mathrm{H}), x\left(a_{1}\right) x^{(i-1)}\left(a_{k}\right) \neq 0$ and we may choose $x(t)$ so that $\operatorname{sgn} x^{(i)}\left(a_{k}\right)=$ $\operatorname{sgn} y\left(a_{k}-\varepsilon\right)$ for $\varepsilon>0$ and small. Let $I$ denote an interval containing $t=a_{1}$ for which $\mathrm{y}(t) \neq 0$ on $I \backslash\left\{a_{1}\right\}$. By Lemma 4 there exists a constant $K>0$ such that if $0<|\lambda|<K$, 


$$
y(t)-\lambda x(t)
$$

has a zero of odd multiplicity on $I$. By Lemma 5 there exists a constant $k, 0<\lambda<K$, for which

$$
z(t)=y(t)-\lambda x(t)
$$

has a simple zero on $\left(a_{k}, a_{k}-\delta\right)$ for $\delta>0$ and small. Then $z(t) \neq \equiv$ by (6) and has the desired properties. If $y(t)$ has a zero at $t=a_{1}$ of even multiplicity, let $x(t)$ be defined by (6) above. The solution $z(t)$ may be obtained by applying Lemma 3 at the point $t=a_{1}$.

For part (iii), we may proceed in a way analogous to the proof of part (ii). In modifying the proof of part (ii), Lemma 1 will play the role of Lemma 5. Since the proof is essentially the same, we omit it here for the sake of brevity. The proof of the theorem is then complete.

The following corollaries are immediate consequences of parts (ii) and (iii) respectively of Theorem 3.

Corollary 1. Suppose $S_{(n-1) j}(\alpha)=\infty$ for some integer $j, 2<$ $j \leqq n-1$. Then for $t \in(\alpha, \infty)$,

$$
S_{(n-2)(j-1)}(t)=S_{(n-3)(j-2)}(t)=\cdots=S_{(n-j+1) 2}(t)=\infty .
$$

CoRollary 2. Suppose $S_{(n-1) j}=\infty$ for some integer $j, 1 \leqq j \leqq$ $n-1$. Let $i$ and $k$ denote integers such that $1 \leqq i \leqq n-2,1 \leqq$ $k \leqq n-i-1$. Then for $t \in[\alpha, \infty)$,

$$
S_{(n-i+1) k}(t) \leqq S_{(n-i) k}(t) .
$$

The following theorem gives a bound on the number of distinct zeros of a nontrivial solution to the right of a zero of multiplicity $l \geqq j+1$.

THEOREM 4. If $1 \leqq j \leqq n-3$, then $S_{(n-1) j}=\infty$ implies for $t \in(\alpha, \infty) S_{(n-j) 1}(t)=\infty$.

Proof. Suppose there exists a nontrivial solution $y(t)$ with $(n-j) \geqq 3$ distinct zeros on $(\alpha, \infty)$ and a zero of order $(j+1)$ in the first position. By Theorem 3(i), we may assume $y(t)$ has distinct zeros at points

$$
t=a<a_{1}<a_{2}<\cdots<a_{n-j-1}
$$

and that there exists an integer $p \geqq 1$ for which $t=a_{p}$ is a zero of odd multiplicity for $y(t)$. Let $c \in[t, \alpha)$ such that $y(c) \neq 0$ and $x(t)$ denote a solution of (1) such that 


$$
\begin{aligned}
x^{(k)}(a) & =0, & k=1, \cdots, j-1 ; \\
x(c) & =0 ; & \\
x\left(a_{k}\right) & =0, & k \neq p .
\end{aligned}
$$

By $(\mathrm{H}), \quad x\left(a_{p}\right) x^{(j)}(a) \neq 0$ and so we may choose $\operatorname{sgn} x^{(j)}(a)$ so that Lemma 5 applies at $t=a$. Since $x\left(a_{p}\right) \neq 0$ by Lemma 4 , we have a constant $K>0$ such that for $0<|\lambda|<K$

$$
y(t)-\lambda x(t)
$$

has a zero close to $t=a_{p}$. Applying Lemma 5, there exists a constant $\lambda_{1} \neq 0$ with $\left|\lambda_{1}\right|<K$ such that the nontrivial solution (by (7))

$$
w(t)=y(t)-\lambda_{1} x(t)
$$

has a zero on $(\alpha, a)$. The solution $w(t)$ has $n-j+1$ distinct zero on $(\alpha, \infty)$ and a zero of order $j$ in the second position. Hence, $S_{(n-j+1) 2}(t)<\infty$ for some $t>\alpha$. This contradicts Corollary 1 if $j>2$ and the hypothesis if $j=2$. The proof of the theorem is complete.

THEOREM 5. If for some integer $j, 1 \leqq j \leqq n-1, S_{(n-1) j}=\infty$ for equation (1), then no oscillatory solution of (1) has a zero of multiplicity $(j+1)$ on $(\alpha, \infty)$.

If $1 \leqq j \leqq n-3$, the theorem follows immediately from Theorem 4. For $j=n-2$, we suppose, to the contrary, that there exists an oscillatory solution $y(t)$ with a zero of order $(n-1)$ at $t=a>\alpha$. Since $S_{(n-1)(n-2)}=\infty$, there exists a point $t=b>a$ for which $y(b)=0$ and $y^{\prime}(b) \neq 0$. Let $c \in(a, \infty)$ for which $y(c) \neq 0$ and $x(t)$ denote a nontrivial solution of $(1)$ satisfying the following $(n-1)$ conditions:

$$
\begin{aligned}
x^{(p)}(a) & =0, \quad p=0,1, \cdots, n-3 ; \\
x(c) & =0 .
\end{aligned}
$$

Note $x(b) x^{(n-2)}(a) \neq 0$ by $(\mathrm{H})$. We choose $\operatorname{sgn} x^{(n-2)}(a)$ so that Lemma 5 applies. Applying Lemma 4 at the point $t=b$, there exists a constant $K>0$ such that if $0<|\lambda|<K$, the solution

$$
y(t)-\lambda x(t)
$$

has a zero close to $t=b$. Applying Lemma 5, there exists a constant $\lambda,|\lambda|<K$, such that

$$
w(t)=y(t)-\lambda x(t)
$$

has a simple zero on $(\alpha, a)$. Hence, the nontrivial soultion $w(t)$ has a $1-(n-2)-1$ distribution of zeros, contradicting Corollary 1 . This completes the proof of the theorem. 
The following theorem provides a separation theorem which is quite useful in the construction of oscillatory solutions.

THEOREM 6. Suppose $S_{(n-1) j}=\infty$ for some integer $j, 1 \leqq j \leqq$ $n-1$, and let $l$ denote an integer such that $1 \leqq l \leqq j-1$. Suppose $y(t)$ is a nontrivial solution of (1) which has a zero of multiplicity $l$ at $t=a \geqq \alpha$. Let $[\gamma, \delta] \subset(a, \infty)$ and suppose $y(t)$ has at least $(j-l-1)$ distinct zeros on $(a, \gamma)$ and at least $(n-j-1)$ distinct zeros on $(\delta, \infty)$. Denote these zeros of $y(t)$ by

$$
a<a_{1}<\cdots<a_{j-l-1}<\gamma<\sigma<b_{1}<\cdots<b_{n-j-1} .
$$

Suppose further that there exists a point $t=c \in(a, \gamma) \cup(\delta, \infty)$ such that $y(t)$ has a zero of odd multiplicity at $t=c$. Then $y(t)$ has only simple zeros on $[\gamma, \delta]$.

Proof. Suppose there exists $\beta \in[\gamma, \delta]$ such that $y(\beta)=y^{\prime}(\beta)=0$. We wish to show that this supposition contradicts $S_{(n-1) j}=\infty$. Obviously, then, $l \geqq 2$. We shall construct a finite sequence of nontrivial solutions $\left\{y_{k}\right\}_{k=0}^{l-1}$ with the properties:

(i) $y_{k}(t)$ has a zero at $t=a$ of order $(l-k)$;

(ii) $y_{k}(t)$ has at least $(j-1)$ zeros counting multiplicities and $(j-l+k)$ distinct zeros on $[a, \gamma)$;

(iii) $y_{k}(t)$ has at least $(n-l+k)$ distinct zeros on $[a, \infty)$;

(iv) $y_{k}(\beta)=y^{\prime}(\beta)=0$.

Having completed the construction, the solution $y_{l-1}(t)$ implies $S_{(n-1) j}(a)<\infty$ contradiction $S_{(n-1) j}=\infty$.

We now proceed with the construction. Define $y_{0}(t) \equiv y(t)$. Let $\eta_{0} \in(a, \infty)$ for which $y\left(\eta_{0}\right) \neq 0$; and $I_{1}$ denote an interval containing $t=c$ for which $y_{0}(t)$ does not vanish on the set $I_{1} \mid\{c\}$. Let $x_{1}(t)$ denote a solution of $(1)$ satisfying the following $(n-1)$ conditions:

$$
\begin{aligned}
& x_{1}^{(p)}(a)=0, \quad p=0,1,2, \cdots, l-2 ; \\
& x_{1}\left(a_{i}\right)=x_{1}\left(b_{m}\right)=0 \text { for all } i \text { and } m \\
& \text { such that } a_{i} \neq c \text { and } b_{m} \neq c ; \\
& x_{1}(\beta)=x_{1}^{\prime}(\beta)=0 ; \\
& x_{1}\left(\eta_{0}\right)=0 .
\end{aligned}
$$

By $(\mathrm{H}), x^{(l-1)}(a) x_{1}(c) \neq 0$ and by Lemma 4 there exists $K_{1}>0$ such that for $|k|<K_{1}$ the solution

$$
y(t)-k x(t)
$$

has a zero on $I_{1}$. By Lemma 1 , there exists a constant $k_{1}$ with $\left|k_{1}\right|<K_{1}$ such that 


$$
y_{1}(t)=y_{0}(t)-k_{1} x_{1}(t)
$$

has a simple zero at $t=c_{1} \in\left(a, a_{1}\right)\left(c_{1} \in(a, \gamma)\right.$ if $\left.l=j-1\right)$ and, of course, a zero at $t=\bar{c} \in I_{1}$. Note further that $y_{1}(t)$ is a nontrivial solution of (1) by (9).

If $l>2$, we proceed with the construction. Let $I_{2}$ denote a closed interval containing $t=c_{1}$ such that $y_{1}(t)$ does not vanish on $I_{2} \mid\left\{c_{1}\right\}$. Let $x_{2}(t)$ denote a solution of $(1)$ satisfying the following $(n-1)$ conditions where $\eta_{1}$ is chosen so that $y_{1}\left(\eta_{1}\right) \neq 0$ :

$$
\begin{aligned}
& x_{2}^{(p)}(a)=0, \quad p=0,1, \cdots, l-3 ; \\
& x_{2}\left(a_{i}\right)=x_{2}\left(b_{m}\right)=0 \text { for all } i \text { and } m \\
& \text { for which } a_{i} \neq c \text { and } b_{m} \neq c ; \\
& x_{2}(\bar{c})=0 ; \\
& x_{2}(\beta)=x_{2}^{\prime}(\beta)=0 ; \\
& x_{2}\left(\eta_{1}\right)=0 .
\end{aligned}
$$

By $(\mathrm{H}), x_{2}^{(l-2)}(a) x_{2}\left(c_{1}\right) \neq 0$ and by Lemma 4 there exists a constant $K_{2}>0$ such that if $|k|<K_{2}$

$$
y_{1}(t)-k x_{2}(t)
$$

has a zero on $I_{2}$. Applying Lemma 1 , there exists a constant $k_{2}$ with $\left|k_{2}\right|<K_{2}$ such that

$$
y_{2}(t)=y_{1}(t)-k_{2} x_{2}(t)
$$

has a zero at $t=c_{2} \in\left(a, c_{1}\right) \cap \bar{I}_{2}$ and a zero at $t=\bar{c}_{1} \in I_{2}$. Note $y_{2}(t)$ is a nontrivial solution by $(\mathbf{1 0})$.

After $(l-1)$ steps, we obtain a nontrivial solution $y_{l-1}(t)$ with the following properties:

$$
\begin{aligned}
& y_{l-1}(a)=0 ; \\
& y_{l-1}^{\prime}(a) \neq 0 ; \\
& y_{l-1}\left(c_{l-1}\right)=0 ; \\
& y_{l-1}\left(\bar{c}_{i}\right)=0, \quad i=1,2, \cdots, l-2 ; \\
& y_{l-1}(\bar{c})=0 ; \\
& y_{l-1}\left(a_{i}\right)=y_{l-1}\left(b_{m}\right)=0 \text { for all } i \text { and } \\
& m \text { for which } a_{i} \neq c \text { and } b_{m} \neq c ;
\end{aligned}
$$$$
y_{l-1}(\beta)=y_{l-1}^{\prime}(\beta)=0 \text {. }
$$

Furthermore,

$$
\begin{aligned}
\alpha & \leqq a<c_{l-1}<\bar{c}_{l-2}<\cdots<\bar{c}_{1}<a_{1}<\cdots \\
& <a_{j-l-1}<\beta<b_{1}<\cdots<b_{n-j-1} .
\end{aligned}
$$

Then $y_{l-1}(t)$ has $(n-1)$ distinct zeros and a double zero at $t=\beta$ 
in the $j$ th position. The contradicts $S_{(n-1) j}=\infty$, and hence no such point $t=\beta$ exists. This completes the proof of the theorem.

We now proceed to the main results of this study. As mentioned before, they give $n$ th-order extension of some of the results of [3], [4], and [5].

Theorem 7. Suppose $S_{(n-1) j}=\infty$ for some integer $j, 1 \leqq j \leqq$ $n-1$. Suppose $y_{l}(t)$ is an oscillatory solution of (1) which has a zero at $t=a>\alpha$ of exact multiplicity $l, 0 \leqq l \leqq j-1$. Then there exists an oscillatory solution $y_{l+1}(t)$ of (1) which has a zero of order at least $(l+1)$ at $t=a$.

To prove the theorem let $\left\{a_{i}\right\}_{i=1}^{\infty}$ denote the consecutive zeros of $y_{l}(t)$ with $a<a_{1}$. Define a sequence of nontrivial solutions $\left\{x_{i}(t)\right\}_{i=1}^{\infty}$ of (1) by the following $(n-1)$ conditions:

$$
\begin{aligned}
& x_{i}^{(p)}(a)=0, \quad p=0,1,2, \cdots, l ; \\
& x_{i}\left(a_{j}\right)=0, \quad k=1,2, \cdots, j-l-1 \text {; } \\
& x_{i}\left(a_{2 i+k}\right)=0, \quad k=1,2, \cdots, n-j-1 \text {. }
\end{aligned}
$$

Suppose first that either $j \neq n-1$ or $l \neq j-1$, and define

$$
\bar{a}= \begin{cases}a_{2 i+1}, & \text { if } j \neq n-1 \\ a_{1}, & \text { if } j=n-1 \text { and } l \neq j-1 .\end{cases}
$$

Consider the intervals $\left(a_{k}, a_{k+1}\right) \subset\left(a_{j-l-1}, a_{2 i+1}\right)$ (where $a_{j-l-1}=a$ if $l=j-1$ and $a_{2 i+1}=\infty$ if $\left.j=n-1\right)$. The claim is that for large $i$ there is at most $(l-1)$ such intervals for which $x_{i}(t)$ does not vanish.

For a proof of this claim, let $\left[a_{k}, a_{k+1}\right] \subset\left(a_{j-l-1}, a_{2 i+1}\right)$ be an interval on which $x_{i}(t)$ does not vanish. By Lemma 2, there exists a nontrivial solution

$$
w_{k}(t)=y_{l}(t)-c_{k} x_{i}(t)
$$

which has a double zero at $t=b_{k} \in\left(a_{k}, a_{k+1}\right)$. Now by Theorem 6 , $w^{\prime}(\bar{a})=0$ and so

$$
c_{k}=\frac{x_{i}^{\prime}(\bar{a})}{y_{l}^{\prime}(\bar{a})} \text {. }
$$

If $\left\{\left[a_{n_{k}}, a_{n_{k}+1}\right]\right\}_{k=1}^{l}$ is a collection as such intervals, then for each $k$ by (13)

$$
c_{n_{k}}=\frac{x_{i}^{\prime}(\bar{a})}{y_{l}^{\prime}(\bar{a})}
$$

and so $w_{n_{1}}(t)$ has $(n-1)$ distinct zeros with a double zero in the $j$ th position. This contradicts the assumption $S_{(n-1) j}=\infty$, and the proof of the claim is complete. 
We return to the proof of the theorem. We now have a sequence $\left\{x_{i}(t)\right\}_{i=1}^{\infty}$ of nontrivial solutions each of which vanishes at $t=a_{k}$, $k=1,2, \cdots, j-l-1$ and a zero of order $(l+1)$ at $t=a$. Furthermore, there is at most $(l-1)$ intervals $\left[a_{k}, a_{k+1}\right] \subset\left(a_{j-l-1}, a_{2 i+1}\right)$ on which $x_{i}(t)$ does not vanish. If $\left\{z_{i}(t)\right\}_{i=1}^{n}$ denotes a fundamental set of solutions for (1), then for each $i$ there exists constants $\left\{c_{i k}\right\}_{k=1}^{n}$ such that

$$
x_{\imath}(t)=\sum_{k=1}^{n} c_{i k} z_{k}(t) .
$$

If we normalize the sequence $\left\{x_{i}(t)\right\}_{i=1}^{\infty}$ by the condition

$$
\sum_{k=1}^{n} c_{i k}^{2}=1
$$

for each $i$, the resulting class of solutions is trivially relatively compact in the solution space. Therefore, there exists a subsequence $\left\{x_{n_{i}}(t)\right\}_{i=1}^{\infty}$ which converges - uniformly on compact sets - to a nontrivial solution $y_{l+1}(t)$ of (1). The zeros of $y_{l+1}(t)$ are limit points of the zeros of the solutions $\left\{x_{n_{i}}(t)\right\}_{i=1}^{\infty}$. It follows from the claim above that $y_{l+1}(t)$ has a zero on $\left[a_{k}, a_{k+1}\right]$ for all but possibly $(l-1)$ such intervals. Hence, $y_{l+1}(t)$ is an oscillatory solution.

To complete the proof of the theorem, we assume $j=n-1$ and $l=j-1=n-2$. We must show that the solution given by

$$
\begin{aligned}
y^{(p)}(a) & =0, \quad p=0,1, \cdots, n-2 \\
y^{(n-1)}(a) & =q \neq 0
\end{aligned}
$$

is oscillatory. If $y(t)$ is nonoscillatory, we may choose $q$ so that $y(t)>0$ for large $t$. By $(\mathrm{H}), y\left(a_{i}\right) \neq 0$ for all $i$. Without loss of generality let $y_{n-2}^{(n-2)}(a)>0$, and let $\left(a_{i}, a_{i+1}\right)$ denote an interval for which $y_{n-2}(t)>0$ (note by assumption $y_{n-2}\left(a_{2}\right)=y_{n-2}\left(a_{i+1}\right)=0$ ). Then by Lemma 2 there exists a constant $\lambda$ and a point $t_{0} \in\left(a_{i}, a_{i+1}\right)$ for which

$$
u(t)=y_{n-2}(t)-\lambda y(t)
$$

has a double zero at $t=t_{0}$. Then

$$
\lambda=\frac{y_{n-2}\left(t_{0}\right)}{y_{n-1}\left(t_{0}\right)}>0 \text {. }
$$

Then

$$
u\left(a_{i}\right) u^{(n-2)}(a)=-\lambda y\left(a_{i}\right) y_{n-2}^{(n-2)}(a)<0 .
$$

It follows that $u(t)$ has a zero of odd multiplicity on $\left(a, a_{i}\right)$ for large $i$. Hence, $y(t)$ vanishes on each interval $\left(a_{i}, a_{i+1}\right)$ for which $y_{n-2}(t)>0$ and $i$ is large. This completes the proof of the theorem. 
Theorem 7 only guarantees the existence of an oscillatory with a zero at $t=a$ of order $j$. The following theorem gives a sharper picture with regard to the number of oscillatory solutions vanishing at $t=a>\alpha$. For $j=n-1$, the added condition $r_{(n-2) 2}=\infty$ is unfortunate. It is not known if $S_{(n-1)(n-1)}=\infty$ implies $r_{(n-2) 2}^{(t)}=\infty$ for large $t$. The condition was used in [4] and played a similar role there.

THEOREM 8. Suppose $S_{(n-1) j}=\infty$ for some integer $j, 1 \leqq j \leqq n-1$. If (1) has an oscillatory solution, then given $a>\alpha$ and an integer $l$, $0 \leqq l \leqq j$, there exists an oscillatory solution $y_{l}(t)$ with a zero at $t=a$ of exact order $l$. Furthermore, every nontrivial linear combination of $y_{l}(t)$ and $y_{l_{-1}}(t)$ is cscillatory except when $l=n-1$. In this case, the added condition $r_{(n-2) 2}=\infty$ implies that every nontrivial linear combination of $y_{n-2}(t)$ is oscillatory.

Theorem 7 assures the existence of $y_{j}(t)$. Given an oscillatory solution $y_{l}(t), 1 \leqq l \leqq j$, with the desired properties, we construct the oscillatory solution $y_{l-1}(t)$ by a method analogous to that used in the proof of Theorem 7. The sequence $\left\{x_{i}(t)\right\}_{i=1}^{\infty}$ will be chosen this time so that

$$
\begin{aligned}
x_{i}^{(p)}(a) & =0, & & p=0,1, \cdots, l-2 ; \\
x_{i}\left(a_{k}\right) & =0, & k & =1, \cdots, j-l, \quad \text { if } \quad l \leqq j-1 ; \\
x_{i}\left(a_{2 i+k}\right) & =0, & k & =1, \cdots, n-j-1, \quad \text { if } j \leqq n-2 ; \\
x_{i}(b) & =0, & &
\end{aligned}
$$

where $\left\{a_{i}\right\}_{i=1}^{\infty}$ denotes the consecutive zeros of $y_{l}(t)$ with $a<a_{1}$ and the point $t=b$ is chosen so that $b \in(\alpha, a)$ and $y_{l}(b) \neq 0$. The method used in Theorem 7 can be used to show that if either $j \neq n-1$ or $l \neq j$, there exist at most $(l-2)$ intervals in $\left(a_{j-l}, a_{2 i+1}\right)$ of the form $\left[a_{i}, a_{i+1}\right]$ for which $x_{i}(t)$ does not have exactly one simple zero on $\left[a_{i}, a_{i+1}\right]$. Furthermore, the sequence $\left\{x_{i}(t)\right\}_{i=1}^{\infty}$ can be normalized by (14) and a corresponding convergent subsequence extracted. The subsequence will converge - uniformly on compact sets - to a nontrivial oscillatory solution $y_{l-1}(t)$, where $y_{l-1}(b)=0$.

We now show that every linear combination of $y_{l}(t)$ and $y_{l-1}(t)$ is oscillatory, i.e., $W\left[y_{l}(t), y_{l-1}(t)\right] \equiv y_{l}(t) y_{l-1}^{\prime}(t)-y_{l-1}(t) y_{l}^{\prime}(t) \neq 0$ for large $t$. Suppose on the contrary that $W\left[y_{l}\left(t_{0}\right), y_{l-1}\left(t_{0}\right)\right]=0$. There then exists a constant $\lambda$ such that

$$
w(t)=y_{l-1}(t)-\lambda y_{l}(t)
$$

has a double zero at $t=t_{0}$. Since $S_{(n-1) j}=\infty$, it follows from (15) and Theorem 6 that $w(t)$ does not change sign on an interval of the 
form $\left[a_{k}, a_{k+1}\right]$ ( $k$ large) if $t_{0}$ is large. Accordingly, $y_{l-1}\left(a_{k}\right) y_{l-1}\left(a_{k+1}\right)>0$ and hence $x_{i}(t)$ has two distinct zeros on $\left(a_{k}, a_{k+1}\right)$ for large enough $i$. This contradicts above statements for $x_{i}(t)$. Therefore, $W\left[y_{l}(t), y_{l-1}(t)\right] \neq 0$ for large values of $t$ and every nontrivial linear combination of $y_{l}(t)$ and $y_{l-1}(t)$ is oscillatory.

To see that $y_{l-1}(t)$ has a zero of exact order $(l-1)$ at $t=a$, note first that if $j=n-1$, then $y_{l-1}(t)$ and $y_{l}(t)$ have $(n-2)$ zeros in common by construction. Since $y_{l-1}(b)=0$ and $y_{l}(b) \neq 0$, the result follows by $(\mathrm{H})$. Now if $j \leqq n-1$ and $l<j, S_{(n-1) j}=$ co implies $y_{l}^{\prime}\left(a_{j-l}\right) y_{l-1}^{\prime}\left(a_{j-l}\right) \neq 0$ and

$$
z(t)=y_{l}^{\prime}\left(a_{j-l}\right) y_{l-1}(t)-y_{l-1}^{\prime}\left(a_{j-l}\right) y_{l}(t)
$$

is oscillatory. Accordingly, Theorem 6 implies $y_{l-1}^{(l-1)}(\alpha) \neq 0$. If $j \leqq n-2$ and $l=j$, then

$$
z(t)=y_{l}^{(l)}(a) y_{l-1}(t)-y_{l-1}^{(l)}(a) y_{l}(t)
$$

is oscillatory, and Theorem 5 implies $y_{l-1}^{(l-1)}(a) \neq 0$.

To complete the proof of the theorem, we need to consider the case $l=j=n-1$. Suppose $y_{n-1}(t)$ has consecutive zeros at $t=a<$ $a_{1}<a_{2}<\cdots<a_{n}<\cdots$, and $y_{n-2}(t)$ denote a nontrivial solution with a zero at $t=a$ of order $(n-2)$ and $y_{n-2}(b)=0$ where $b>a$ is chosen so that $y_{n-1}(b) \neq 0$. Since $y_{n-2}(t)$ and $y_{n-1}(t)$ have $(n-2)$ zeros in common at $t=a,(\mathrm{H})$ implies $y_{n-2}^{(n-2)}(a) \neq 0$. Suppose $\left[a_{k}, a_{k+1}\right], b<a_{k}$, is an interval for which $y_{n-2}(t) \neq 0$. Lemma 2 and $(\mathrm{H})$ imply for some $t_{0} \in\left(a_{k}, a_{k+1}\right)$ the solution

$$
w(t)=y_{n-2}(t)-\left(\frac{y_{n-1}\left(t_{0}\right)}{y_{n-2}\left(t_{0}\right)}\right) y_{n-1}(t)
$$

has a double zero at $t=t_{0}$. By Theorem 6 ,

$$
0<w(b) w^{(n-2)}(a)=-\left(\frac{y_{n-1}\left(t_{0}\right)}{y_{n-2}\left(t_{0}\right)}\right) y_{n-1}(b) y_{n-2}^{(n-2)}(a) .
$$

Hence $y_{n-2}(t)$ vanishes on each interval $\left(a_{\imath}, a_{\imath+1}\right)$ for which $\operatorname{sgn} y_{n-1}^{\prime}\left(a_{i}\right) y_{n-2}\left(a_{\imath}\right)=\operatorname{sgn} y_{n-1}(b) y_{n-2}^{(n-2)}(a)$. Therefore, $y_{n-2}(t)$ is oscillatory. Finally, the last statement in Theorem 7 follows immediately from Lemma 2. The proof of the theorem is then complete.

\section{REFERENCES}

1. J. H. Barrett, Oscillation theory of ordinary differential equations, Associated Western Universities Differential Equations Symposium, Boulder, Colorado, Summer 1967. 2. J. M. Dolan, Oscillation behavior of solutions of linear differential equations of third-order, Ph. D. dissertation, The University of Tennessee, 1967.

3. Maurice Hanan, Oscillation criteria for third-order linear differential equations, Pacific J. Math., 11 (1961), 919-944. 
4. M. S. Keener, On oscillatory solutions of certain fourth-order linear differential equations, SIAM. J. of Math. Anal., (to appear).

5. Walter Leighton and Zeev Nehari, On the oscillations of solutions of self-adjoint linear differential equations of the fourth-order, Trans. Amer. Math. Soc., 89 (2), (1958), 325-377.

6. A. C. Peterson, A theorem of Alieu, Proc. Amer. Math. Soc., 23 (2), (1969), 364-366. 7. - On the ordering of multi-point boundary value functions, Canad. Math. Bull., 13 (4), (1970), 507-513.

8. T. L. Sherman, Conjugate points and simple zeros for ordinary linear differential equations, Trans. Amer. Math. Soc., 146 (1969), 397-411.

Received November 24, 1972 and in revised form April 30, 1973.

OKLAhoma State UNiversity 


\section{PACIFIC JOURNAL OF MATHEMATICS}

\section{EDITORS}

RICHARD ARENS (Managing Editor)

University of California

Los Angeles, California 90024

R. A. BeAumont

University of Washington

Seattle, Washington 98105
J. DugundJI*

Department of Mathematics

University of Southern California

Los Angeles, California 90007

D. Gilbarg and J. Milgram

Stanford University

Stanford, California 94305

\section{ASSOCIATE EDITORS}

E. F. BECKENBACH

B. H. NEUMANN

F. WOLF

K. YoSHIDA

\section{SUPPORTING INSTITUTIONS}

UNIVERSITY OF BRITISH COLUMBIA
CALIFORNIA INSTITUTE OF TECHNOLOGY
UNIVERSITY OF CALIFORNIA
MONTANA STATE UNIVERSITY
UNIVERSITY OF NEVADA
NEW MEXICO STATE UNIVERSITY
OREGON STATE UNIVERSITY
UNIVERSITY OF OREGON
OSAKA UNIVERSITY

UNIVERSITY OF BRITISH COLUMBIA CALIFORNIA INSTITUTE OF TECHNOLOGY UNIVERSITY OF CALIFORNIA MONTANA STATE UNIVERSITY NEW MEXICO STATE UNIVERSITY UNIVERSITY OF OREGON OSAKA UNIVERSITY
UNIVERSITY OF SOUTHERN CALIFORNIA STANFORD UNIVERSITY UNIVERSITY OF TOKYO UNIVERSITY OF UTAH WASHINGTON STATE UNIVERSITY UNIVERSITY OF WASHINGTON AMERICAN MATHEMATICAL SOCIETY NAVAL WEAPONS CENTER

* C. R. DePrima California Institute of Technology, Pasadena, CA 91109, will replace J. Dugundji until August 1974. 


\section{Pacific Journal of Mathematics}

\section{Vol. 51, No. $1 \quad$ November, 1974}

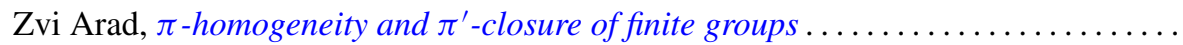

Ivan Baggs, A connected Hausdorff space which is not contained in a maximal

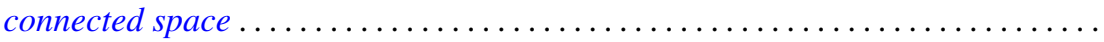

Eric Bedford, The Dirichlet problem for some overdetermined systems on the unit ball in $C^{n}$

R. H. Bing, Woodrow Wilson Bledsoe and R. Daniel Mauldin, Sets generated by

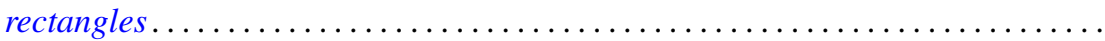

Carlo Cecchini and Alessandro Figà-Talamanca, Projections of uniqueness for

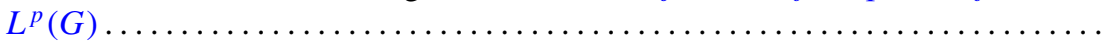

Gokulananda Das and Ram N. Mohapatra, The non absolute Nörlund summability of Fourier series .

Frank Rimi DeMeyer, On separable polynomials over a commutative ring ........ Richard Detmer, Sets which are tame in arcs in $E^{3} \ldots \ldots \ldots \ldots \ldots \ldots \ldots \ldots$

William Erb Dietrich, Ideals in convolution algebras on Abelian groups ..........

Bryce L. Elkins, A Galois theory for linear topological rings .................

William Alan Feldman, A characterization of the topology of compact convergence on $C(X)$.

Hillel Halkin Gershenson, A problem in compact Lie groups and framed cobordism

Samuel R. Gordon, Associators in simple algebras.

Marvin J. Greenberg, Strictly local solutions of Diophantine equations

Jon Craig Helton, Product integrals and inverses in normed rings . . . . . . . . . . . .

Domingo Antonio Herrero, Inner functions under uniform topology . . .

Jerry Alan Johnson, Lipschitz spaces .

Marvin Stanford Keener, Oscillatory solutions and multi-point boundary value

functions for certain nth-order linear ordinary differential equations.

John Cronan Kieffer, A simple proof of the Moy-Perez generalization of the

Shannon-McMillan theorem .......................

Joong Ho Kim, Power invariant rings

Gangaram S. Ladde and V. Lakshmikantham, On flow-invariant sets .

Roger T. Lewis, Oscillation and nonoscillation criteria for some self-adjoint even

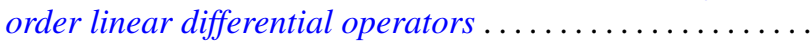

Jürg Thomas Marti, On the existence of support points of solid convex sets ..

John Rowlay Martin, Determining knot types from diagrams of knots . .

James Jerome Metzger, Local ideals in a topological algebra of entire functions

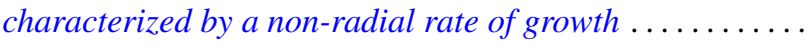

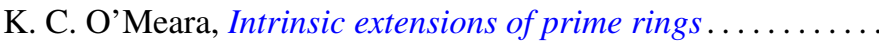

Stanley Poreda, A note on the continuity of best polynomial approximations ..

Robert John Sacker, Asymptotic approach to periodic orbits and local prolongations

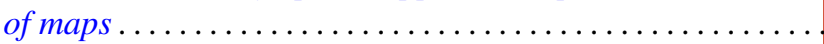

Eric Peter Smith, The Garabedian function of an arbitrary compact set . .

Arne Stray, Pointwise bounded approximation by functions satisfying a side condition

John St. Clair Werth, Jr., Maximal pure subgroups of torsion complete abelian

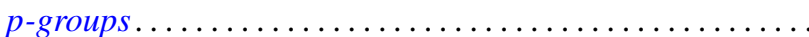

